\title{
Small-scale swirl events in the quiet Sun chromosphere ${ }^{\star}$
}

\author{
S. Wedemeyer-Böhm ${ }^{1,2, \star \star}$ and L. Rouppe van der Voort ${ }^{1}$ \\ 1 Institute of Theoretical Astrophysics, University of Oslo, PO Box 1029 Blindern, 0315 Oslo, Norway \\ e-mail: sven.wedemeyer-bohm@astro.uio.no \\ 2 Center of Mathematics for Applications, University of Oslo, Box 1053 Blindern, 0316 Oslo, Norway
}

Received 30 September 2009 / Accepted 14 October 2009

\begin{abstract}
Context. Recent progress in instrumentation enables solar observations with high resolution simultaneously in the spatial, temporal, and spectral domains.

Aims. We use such high-resolution observations to study small-scale structures and dynamics in the chromosphere of the quiet Sun. Methods. We analyse time series of spectral scans through the Ca II $854.2 \mathrm{~nm}$ spectral line obtained with the CRISP instrument at the Swedish 1-m Solar Telescope. The targets are quiet Sun regions inside coronal holes close to disc-centre.

Results. The line core maps exhibit relatively few fibrils compared to what is normally observed in quiet Sun regions outside coronal holes. The time series show a chaotic and dynamic scene that includes spatially confined "swirl" events. These events feature dark and bright rotating patches, which can consist of arcs, spiral arms, rings or ring fragments. The width of the fragments typically appears to be of the order of only 0.2 , which is close to the effective spatial resolution. They exhibit Doppler shifts of -2 to $-4 \mathrm{~km} \mathrm{~s}^{-1}$ but sometimes up to $-7 \mathrm{~km} \mathrm{~s}^{-1}$, indicating fast upflows. The diameter of a swirl is usually of the order of $2^{\prime \prime}$. At the location of these swirls, the line wing and wide-band maps show close groups of photospheric bright points that move with respect to each other. Conclusions. A likely explanation is that the relative motion of the bright points twists the associated magnetic field in the chromosphere above. Plasma or propagating waves may then spiral upwards guided by the magnetic flux structure, thereby producing the observed intensity signature of Doppler-shifted ring fragments.
\end{abstract}

Key words. Sun: atmosphere - Sun: chromosphere - Sun: magnetic fields

\section{Introduction}

Advances in observational performance have revolutionised our understanding of the solar chromosphere over the last years. It is in particular the combination of high spectral, temporal, and spatial resolution that let us discover details and phenomena hitherto unaccessible. Our picture of the solar atmosphere changed from a static plane-parallel stratification to a very complex compound of intricately coupled dynamic domains (see the recent reviews by e.g., Schrijver 2001; Judge 2006; Rutten 2006, 2007; Wedemeyer-Böhm et al. 2009). And yet many details concerning the chromosphere and its connection to the layers above and below remain elusive. The progress in our understanding is certainly hampered by the complexity and accessibility of currently available diagnostics probing that atmospheric layer. Among them are the spectral lines of $\mathrm{Ca}$ II, which are formed over a very extended height range in the atmosphere. Only the line cores truly originate from above the photosphere. Observing the line core exclusively therefore requires filters with a very narrow transmitted wavelength range, as the width of the line cores is only of the order of 100 pm or less.

Considering the spectral, temporal, and spatial domains, instrumental limitations enforce compromises which until recently only allowed high resolution in one or two of the domains, at the cost of the remaining one(s). This situation has now improved substantially with the installation of fast

* The movie is only available in electronic form at http: //www . aanda.org

$\star \star$ Marie Curie Intra-European Fellow of the European Commission. two-dimensional spectropolarimetric imagers at solar telescopes with large aperture. Examples are the IBIS instrument (Cavallini 2006) at the Dunn Solar Telescope, CRISP (Scharmer et al. 2008) at the Swedish Solar Telescope (SST), and the Göttingen Fabry-Pérot (Puschmann et al. 2006) at the German Vacuum Tower Telescope. Achieving high resolution simultaneously in all these three domains is like opening a new observing window on the chromosphere. Here we report on the discovery of small but fast rotating swirls in the chromosphere. There are quite a few examples known of rotating or vortex-like motions on the Sun: on large scales in the form of rotating sunspots (e.g., Brown et al. 2003), on smaller scales like vortices in the photospheric granulation (Brandt et al. 1988), and on the smallest scales in the form of whirlpool motion by magnetic bright points in the intergranular lanes (Bonet et al. 2008). This rotating motion receives considerable interest since these photospheric motions have a profound effect on the outer-atmospheric magnetic fields as they have their roots in the photoshere. The stresses that are built up through rotation of magnetic fields are linked to the onset of solar flares and coronal heating (e.g., Parker 1983).

After this introduction we describe the observations in Sect. 2, which are analysed in Sect. 3. A discussion and conclusions are given in Sect. 4.

\section{Observations}

The observations were obtained with the CRisp Imaging SpectroPolarimeter (CRISP, Scharmer et al. 2008) installed at the Swedish 1-m Solar Telescope (SST, Scharmer et al. 2003) on 
Table 1. Ca II 854.2 line scan sequences from June 2008.

\begin{tabular}{ccccccc}
\hline \hline $\begin{array}{c}\text { Data set } \\
\text { (date) }\end{array}$ & $\mu$ & $\begin{array}{c}\lambda \\
{[\mathrm{pm}]}\end{array}$ & $\begin{array}{c}(\Delta \lambda)_{\text {core }}{ }^{a} \\
{[\mathrm{pm}]}\end{array}$ & $\begin{array}{c}(\Delta \lambda)_{\text {wing }}{ }^{b} \\
{[\mathrm{pm}]}\end{array}$ & $\begin{array}{c}\Delta t \\
{[\mathrm{~s}]}\end{array}$ & $\begin{array}{c}\text { dur. } \\
{[\mathrm{min}]}\end{array}$ \\
\hline $1(13$ th) & 0.92 & $-92.0-+19.4$ & 4.8 & 4.8 & 9 & 33 \\
$2(15$ th) & 0.99 & $-193.9-+193.9$ & 9.7 & 19.4 & 11 & 53 \\
\hline
\end{tabular}

${ }^{a}$ Wavelength sampling for $-70 \mathrm{pm}<\lambda<70 \mathrm{pm}$ (core); ${ }^{b}$ wavelength sampling for $\lambda>70 \mathrm{pm}, \lambda<-70 \mathrm{pm}$ (wings).

La Palma (Spain). CRISP is a spectropolarimeter that includes a dual Fabry-Pérot interferometer and is capable of fast wavelength tuning ( $\$ 50 \mathrm{~ms})$, which makes it ideally suited for imaging the dynamic chromosphere. We observed the Ca II $854.2 \mathrm{~nm}$ line (filter transmission FWHM $11.1 \mathrm{pm}$ ) and analyse two time sequences from two days in 2008, targeting equatorial coronal holes close to disc center (field of view, FOV $\approx 71^{\prime \prime} \times 71^{\prime \prime}$ ). All data sets are complemented with wide-band images from the CRISP prefilter $(F W H M=0.93 \mathrm{~nm}$ centered on $854.2 \mathrm{~nm})$. On June 15th 2008, a series of $\mathrm{H} \alpha$ images was recorded directly after the $\mathrm{Ca}$ scan at the same position. More details on the data are presented in Table 1. With the use of adaptive optics combined with Multi-Object Multi-Frame Blind Deconvolution (MOMFBD, van Noort et al. 2005) image restoration, the time sequences are of excellent quality, achieving a spatial resolution close to the diffraction limit $\left(\lambda / D=0{ }^{\prime} 18\right.$ at $\left.854.2 \mathrm{~nm}\right)$ for the majority of the images. For more details on the observing and reduction procedures we refer to Scharmer et al. (2008), and Rouppe van der Voort et al. (2009). In the latter publication, the 15-Jun.-2008 data set has been analysed in the context of the connection between rapid blue-shifted excursions and type II spicules. Doppler shifts are determined as the shift of the line core with respect to the undisturbed line core wavelength of $854.2 \mathrm{~nm}$

\section{Analysis}

Atmospheric structure. Traditionally, the prime diagnostic for the chromosphere has been the $\mathrm{H} \alpha$ line. The IBIS observations by Cauzzi et al. (2008) and Vecchio et al. (2009) have demonstrated that the Ca II $854.2 \mathrm{~nm}$ spectral line serves as an excellent alternative chromospheric diagnostic with the advantage of more straightforward interpretation as compared to $\mathrm{H} \alpha$ (see e.g., Leenaarts et al. 2009). Cauzzi et al. (2008) find that Ca II $854.2 \mathrm{~nm}$ line core intensity maps reveal a fibrilar structure similar to what is well known from $\mathrm{H} \alpha$ observations, and that fibrils cover a large fraction of the field of view even in more quiet regions. Next to the fibrils, which outline the magnetic field in the chromosphere, a dynamic pattern produced by propagating shock waves (see e.g. Wedemeyer-Böhm et al. 2009) is present. In contrast, our CRISP line core maps, which were obtained in coronal holes, exhibit only few fibrils, which cover only a limited fraction of the field of view. The magnetic field configuration in- and outside coronal holes is very different (see e.g. Cranmer 2009, and references therein), which has direct consequences for the morphology of the Ca II $854.2 \mathrm{~nm}$ line core maps. Similarly, the $\mathrm{H} \alpha$ images directly taken after data set 2 show a small number of fibrils.

The scene of apparent magnetic quiescence is consistent with the atmosphere in the layers below, which show no pronounced magnetic network. The FOV in the wide-band is characterised by granulation with bright points (BPs) in the intergranular lanes (see the upper row in Fig. 1). These BPs are known to be connected to magnetic field concentrations and are routinely used as proxy for the magnetic field. The wide-band image essentially yields the continuum intensity and thus effectively maps the lower photosphere. The map in the blue line wing at $\Delta \lambda=-92.1 \mathrm{pm}$ is clearly dominated by the reversed granulation pattern, which is formed in the middle photosphere (Leenaarts \& Wedemeyer-Böhm 2005).

Swirls. The line core time sequences show small regions with dark and bright rotating patches, which can consist of arcs, spiral arms, rings or ring fragments. In Fig. 1 and the movie in the online material, we present a prominent example with a ring. The width of the ring is mostly $\sim 0$.' 2 , whereas the diameter is $\sim 2^{\prime \prime}$. The pattern can also be seen in the blue line wing at $\Delta \lambda=-53.3 \mathrm{pm}$ and sometimes down to $\sim-70 \mathrm{pm}$, which is formed in the middle/upper photosphere. At exactly the same position a bright ring-like counterpart appears in the red wing. The size of the pattern stays similar throughout this wavelength range. The line core is blue-shifted at positions in the ring, corresponding to upward (LOS) velocities typically of the order of -2 to $\sim-4 \mathrm{~km} \mathrm{~s}^{-1}$. The swirl exhibits a small ring fragment with high Doppler shift at a position of $\left[x \approx 1^{\prime \prime} .0, y \approx 0.0\right]$ at $t=0 \mathrm{~s}$ that appears to rotate clockwise (see Fig. 1). The Doppler shift of that line core feature is initially $-4.3 \mathrm{~km} \mathrm{~s}^{-1}$ and exceeds $\sim-5 \mathrm{~km} \mathrm{~s}^{-1}$ in the subsequent frames. The feature appears to have rotated by $\sim 90$ degrees after roughly a minute. The centre of rotation is located in the central brightening visible in the line core maps, roughly coinciding with a BP group in the wide-band images. An estimate of the speed of the feature along its circular track yields approximate values of the order of $\sim 10 \mathrm{~km} \mathrm{~s}^{-1}$. However, the feature evolves in time and cannot be tracked as reliably as wide-band BPs. The imprint of ring fragments is also seen in spectral line depth, being deepest where the line core intensity is lowest, and in the line width, measured at constant count levels. The latter is slightly increased at the rim of the ring.

Another interesting aspect concerns the bright feature, which can be seen at a position of $[x \approx-1 . .5, y \approx 0.5]$ in the line core map at $t=90 \mathrm{~s}$ (see Fig. 1). It is located on a bright ring fragment just outside the dark ring and can be seen throughout the displayed sequence. The feature starts off rather faintly but increases in brightness as it follows the clockwise rotation. It is accompanied by a strong Doppler shift which grows in time to up to $-6.9 \mathrm{~km} \mathrm{~s}^{-1}$ before it quickly fades away after $t=90 \mathrm{~s}$. The line wing and wide-band maps show nothing special in these moments so that this event is likely to be confined to the layers above the photosphere. More of these features appear during the swirl event.

We interpret the blue-shift and the clockwise rotation as plasma spiraling upwards in a funnel-like magnetic field structure with a diameter of $\sim 2^{\prime \prime}$. It may be due to propagating wave fronts, which are guided by the magnetic field. The short-lived bright features with high Doppler shift may be explained as plasma which is accelerated and then ejected upwards and away from the swirl at large velocity.

Statistics. Swirls are frequently observed in both our coronal hole data sets. For a measure of the swirl statistics, we performed a thorough inspection of dataset 1 . We found 10 clear examples, including the one discussed above. In addition, we found 10 cases which feature (minor) swirl action, arcs, spirals and/or ring fragments for a short time span but not as strikingly and lastingly as in the clear detections mentioned before. Finally, there are another 16 potential but less reliable 


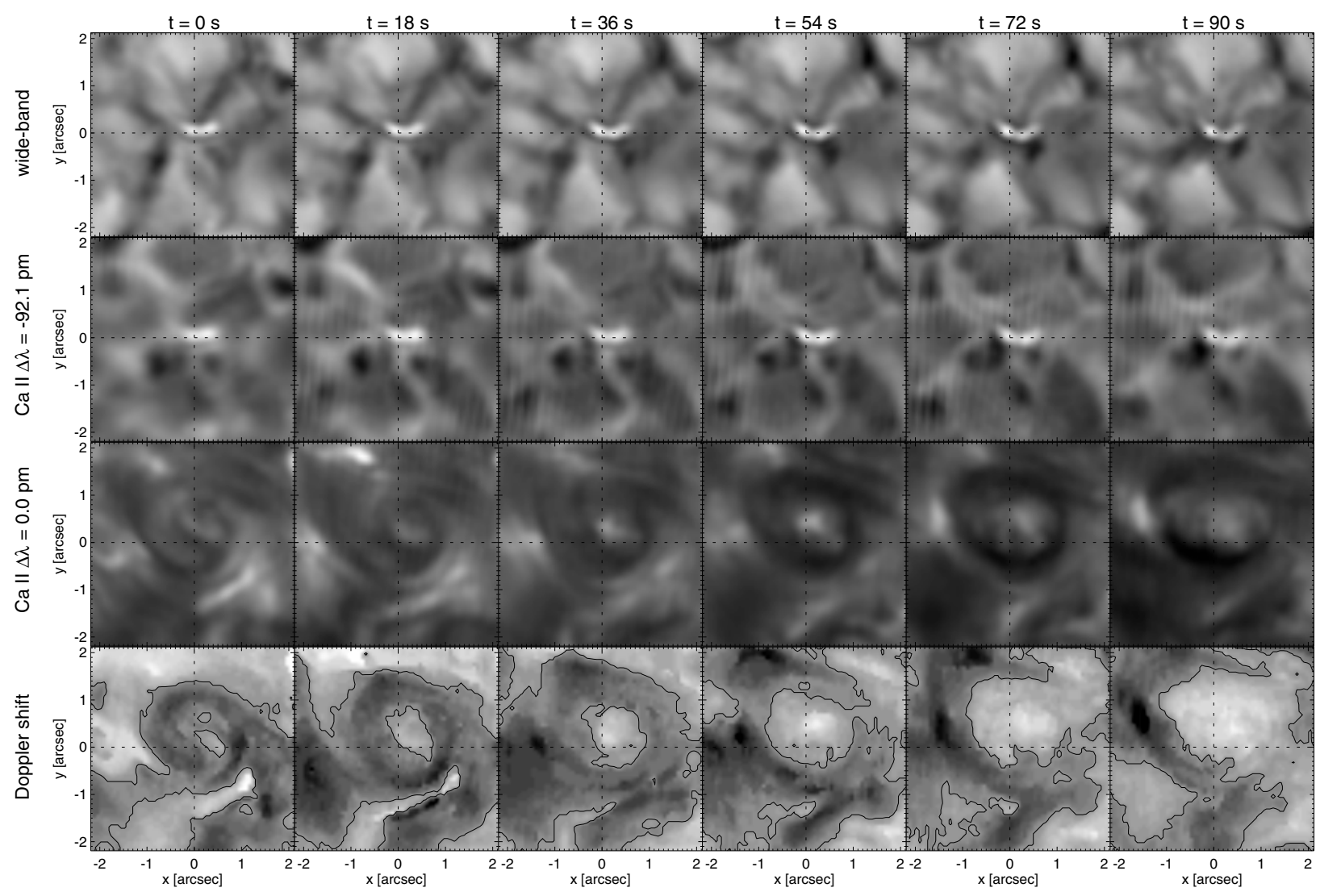

Fig. 1. Temporal evolution of a swirl event as seen in close-ups of intensity maps in the wide-band (top row), Ca line wing (upper middle), Ca line core (lower middle), and Doppler shift (bottom). The columns from left to right show every other time step $(\Delta t=18 \mathrm{~s})$. The black contours in the bottom row mark a zero Doppler shift. The grey scale of the Doppler shift is from -5.5 to $+5.5 \mathrm{~km} \mathrm{~s}^{-1}$ with negative values corresponding to blueshifts and thus upflows. The temporal evolution is presented in a movie provided as online material.

detections. Unambiguously discerning a faint swirl imprint requires high data quality over a sufficiently long period. That can be hampered by variation in the observation conditions and resulting low contrast but also by the presence of fibrils. It is therefore difficult to determine reliable event durations. We see swirls that can only be identified for a few consecutive time steps and other swirls that last for minutes and/or reappear at the same location later in the time series. In the region presented in Fig. 1, swirling motions are detected for about 12 min of which the first $8 \mathrm{~min}$ are very clear. A conservative estimate of the frequency of swirl event occurrence yields a value of 0.24 swirls $\mathrm{min}^{-1} \mathrm{arcmin}^{-2}$ based on the clear detections. It increases to 0.48 swirls $\mathrm{min}^{-1} \operatorname{arcmin}^{-2}$ when taking into account the potential detections, too.

The details of the swirl pattern in the atmosphere above varies from case to case. The rings in the line core maps have radii of typically 1 arcsec, although there are examples with radii down to $0 .{ }^{\prime} 4-0 .{ }^{\prime} 6$ and up to $11^{\prime \prime} 5$. The line core rings can be seen during most of the event, while the Doppler shift signature can fade away and reappear again. We also find some cases for which spiral arms are visible instead of or in addition to a roughly concentric ring (see Figs. 3b,c). The inner parts of such spiral arms are found 0.' 4 to 1'.0 away from the BP, while the outer tip, which is leading the rotation, can be traced up to a distance of $\sim 2^{\prime \prime}$. Dopplergrams show that the blue-shift of the line core tends to increase along the dark spiral arms outwards and can reach upward velocities of up to $\sim 7 \mathrm{~km} \mathrm{~s}^{-1}$. In some examples it appears as if plasma is accelerated and then ejected away from the central BP group.
Based on the swirls identified, there does not seem to be a preferred direction of rotation. Also the location within the observed coronal hole appears to be random.

Photospheric bright point groups. The line wing and wideband images, which map the middle and low photosphere, show small groups of photospheric BPs at the locations of all detected swirls. These photospheric BPs move vigorously along the intergranular lanes and the brights rims of the reversed granulation pattern and below, indicating that they are buffeted by the lateral granular flows. In Fig. 2 we show the movement of the photospheric BPs for the example presented in Fig. 1. A bright elongated structure is seen in the initial wide-band image (upper row) at the location of the swirl. The brightening appears to consist of at least 2 (unresolved) BPs. By using a feature tracking algorithm, we find that the initial bright structure breaks apart into at least three individual BPs with separations of only a few tenths of an arcsecond. The BPs move with average speeds between 1.5 and $2.0 \mathrm{~km} \mathrm{~s}^{-1}$ for the example in Fig. 2. The relative motion pattern is subject to the local granular flow field and thus varies from case to case. Generally, BPs related to swirls are found to have lateral speeds between 0.7 and $2.0 \mathrm{~km} \mathrm{~s}^{-1}$, which is consistent with the expected advection of the BPs with the granular flow field. In Fig. 2, two BPs seem to cross their paths, which has consequences for the magnetic field topology in the chromosphere above. The other swirls also show close encounters of BPs. Although it is sometimes difficult to resolve all individual components, an apparently chaotic relative motion of BPs seems to be common below swirl regions. 


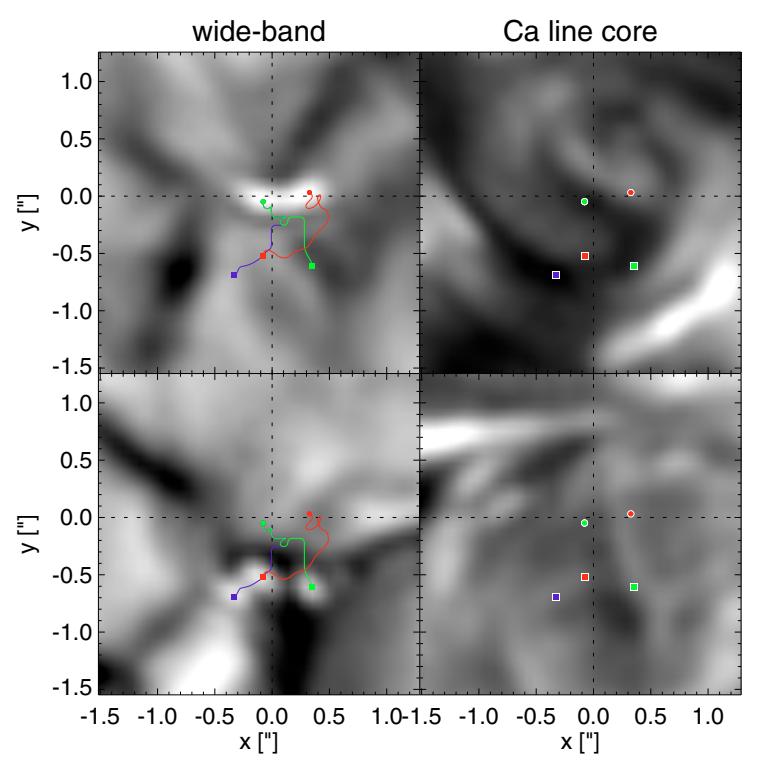

Fig. 2. Motion of photospheric bright points seen in the wide-band images (left column) at the position of a selected swirl (seen in the Ca line core images, right column). The two rows show two images with a separation of $10 \mathrm{~min}$. The initial positions of the BP tracks are marked with circles, the final ones with squares.
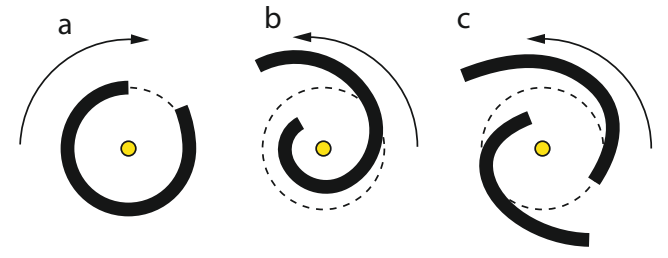

Fig. 3. Schematic structure of swirl events. The radius of the dotted circle is typically of the order of $1^{\prime \prime}$.

\section{Discussion and conclusions}

At first glance, the swirls in the coronal hole are reminiscent of the convectively driven vortex flows reported by Bonet et al. (2008). In a high-resolution SST G-band filtergram time sequence, they found examples of groups of BPs displaying clear "whirlpool" motion. Bonet et al. associate the photospheric whirlpools with vigorous downdrafts at the vertices of intergranular lanes that have been predicted by convection models. The G-band BPs act as flow tracers which follow spiral trajectories when they are engulfed by the downdrafts. The size of these whirlpools is less than an arcsecond. Contrastingly, the photospheric BPs associated with the chromospheric swirls in our data do not follow clear spiral trajectories. It is therefore unclear if photospheric whirlpools and chromospheric swirls are connected.

Our wide-band images usually show a small number of photospheric BPs that move in relation to each other, buffeted by the lateral granular flows at the top of the convection zone. Being confined to the intergranular lanes, the BPs can eventually come close and even move past each other very closely. Our interpretation is that these close encounters result in a deformation and twist of the magnetic field continuing above the BPs. While tending to unwind the build-up stresses, gas may spiral upwards, producing the observed intensity signature of Doppler-shifted ring fragments.
Such a process may be impeded by the presence of a stronger magnetic field component in the chromosphere in the form of a "magnetic canopy" (e.g., Schrijver \& Title 2003) as it exists above quiet Sun regions outside coronal holes. Swirls are therefore expected to be significantly less frequent in quiet Sun regions outside coronal holes, which have a high fibril coverage. Quantifying this assumption is unfortunately difficult as the detection of swirls in $\mathrm{Ca}$ line core maps is complicated by the presence of fibrils. Events ongoing in the layer just below the fibrils could be obscured by the horizontal component of the magnetic field (Vecchio et al. 2009).

The exact cause for the swirl phenomenon remains to be found. The magnetic fields, which are supposedly responsible for forcing the rotating plasma or propagating waves on ring-like or spiral trajectories, are not directly detected in our CRISP observations. However, all our detected swirls show a small group of BPs in the centre, implying a causal connection.

We interpret these chromospheric swirl motions and associated BP motions as a direct indication of upper-atmospheric magnetic field twisting and braiding as a result of convective buffeting of magnetic footpoints. This mechanism is one of the prime candidates for coronal heating (Parker 1988).

Acknowledgements. This study made use of the CRISPEX data explorer programmed by Gregal Vissers. The Swedish 1-m Solar Telescope is operated on the island of La Palma by the Institute for Solar Physics of the Royal Swedish Academy of Sciences in the Spanish Observatorio del Roque de los Muchachos of the Instituto de Astrofísica de Canarias. S.W.B. acknowledges support through a Marie Curie Intra-European Fellowship of the European Commission (FP6-2005-Mobility-5, Proposal No. 042049). This research was supported by the Research Council of Norway through grant 170935/V30.

\section{References}

Bonet, J. A., Márquez, I., Sánchez Almeida, J., Cabello, I., \& Domingo, V. 2008, ApJ, 687, L131

Brandt, P. N., Scharmer, G. B., Ferguson, S., Shine, R. A., \& Tarbell, T. D. 1988, Nature, 335, 238

Brown, D. S., Nightingale, R. W., Alexander, D., et al. 2003, Sol. Phys., 216, 79

Cauzzi, G., Reardon, K. P., Uitenbroek, H., et al. 2008, A\&A, 480, 515

Cavallini, F. 2006, Sol. Phys., 236, 415

Cranmer, S. R. 2009, Living Rev. Solar Phys., 6, 3

Judge, P. 2006, in Solar MHD Theory and Observations: A High Spatial Resolution Perspective, ed. J. Leibacher, R. F. Stein, \& H. Uitenbroek, ASP Conf. Ser., 354, 259

Leenaarts, J., \& Wedemeyer-Böhm, S. 2005, A\&A, 431, 687

Leenaarts, J., Carlsson, M., Hansteen, V., \& Rouppe van der Voort, L. 2009, ApJ, 694, L128

Parker, E. N. 1983, ApJ, 264, 642

Parker, E. N. 1988, ApJ, 330, 474

Puschmann, K. G., Kneer, F., Seelemann, T., \& Wittmann, A. D. 2006, A\&A, 451,1151

Rouppe van der Voort, L., Leenaarts, J., de Pontieu, B., Carlsson, M., \& Vissers, G. 2009, ApJ, 705, 272

Rutten, R. J. 2006, in Solar MHD Theory and Observations: A High Spatial Resolution Perspective, ed. J. Leibacher, R. F. Stein, \& H. Uitenbroek, ASP Conf. Ser., 354, 276

Rutten, R. J. 2007, in The Physics of Chromospheric Plasmas, ed. P. Heinzel, I. Dorotovič, \& R. J. Rutten, ASP Conf. Ser., 368, 27

Scharmer, G. B., Bjelksjo, K., Korhonen, T. K., Lindberg, B., \& Petterson, B. 2003, in SPIE Conf. Ser. 4853, ed. S. L. Keil, \& S. V. Avakyan, 341

Scharmer, G. B., Narayan, G., Hillberg, T., et al. 2008, ApJ, 689, L69

Schrijver, C. J. 2001, in 11th Cambridge Workshop on Cool Stars, Stellar Systems and the Sun, ed. R. J. Garcia Lopez, R. Rebolo, \& M. R. Zapaterio Osorio, ASP Conf. Ser., 223, 131

Schrijver, C. J., \& Title, A. M. 2003, 597, L165

van Noort, M., Rouppe van der Voort, L., \& Löfdahl, M. G. 2005, Sol. Phys., 228,191

Vecchio, A., Cauzzi, G., \& Reardon, K. P. 2009, A\&A, 494, 269

Wedemeyer-Böhm, S., Lagg, A., \& Nordlund, Å. 2009, Space Sci. Rev., 144, 317 